\title{
A Study of Thermal Behaviour of HTS Devices at Alternating Current
}

\author{
V. Z. Manusov, D. O. Krjukov
}

Departement of Power Supply Systems, Novosibirsk State Technical University, Russian Federation

\begin{tabular}{l} 
Article Info \\
\hline Article history: \\
Received Jan 5, 2018 \\
Revised Mar 10, 2018 \\
Accepted Mar 27, 2018 \\
\hline
\end{tabular}

Keyword:

Automatic reclosing

Short-circuit current limitation

Superconducting transformer

Superconducting winding

Voltage-current characteristics

Windings overheating

\begin{abstract}
The paper presents a study on thermal behavior of a coil made of a hightemperature supercon-ducting tape representing operation of a superconducting device (for example, a transformer). Based on the results of a physical experiment, a mathematical model of superconducting coil operation under short circuit conditions at the load side was developed. Regimes of overheating by variable short circuit currents were investigated. In this case, short circuit current amplitudes ex-ceeded a critical current of a superconductor, and coil parameters (e.g. an average nonlinearity parameter of a current-voltage characteristic, a superconductor length, and others) were varied. Permissible overheating for a coil with the possibility of its return into the superconducting state after emergency conditions in a dead-time period of automatic reclosing was considered. A crite-rion for return of a superconducting device into the superconducting state within a dead-time period of automatic reclosing has been obtained.
\end{abstract}

Copyright $@ 2018$ Institute of Advanced Engineering and Science. All rights reserved.

\section{Corresponding Author:}

D. O. Krjukov,

Departement of Power Supply Systems,

Novosibirsk State Technical University,

20, Karl Marx prospekt, Novosibirsk, 630073, Russian Federation.

Email: fire-paladin@mail.ru

\section{INTRODUCTION}

Today, more than ever before, power engineering industry in Russia needs innovations in the power equipment sector. It is influenced by not only progressive ageing of existing equipment used for efficient power transmission and transformation, but also obsolescence of the paradigm of power engineering management. In the age of up-to-date technological decisions in the power engineering industry with a glance to global striving to energy saving and increasing economic efficiency of existing equipment, the problem of implementing superconducting technologies has arisen again.

A rising tide of interest to superconductivity can be explained, in general, by the decrease of prices for superconducting wire manufacturing. At the present time, the price for the 2nd generation hightemperature superconducting (HTS) wire is about $400 \$ / \mathrm{kA} * \mathrm{~m}$. At the same time, material cost for production of, for example, superconducting tapes of the 2 nd generation equals to about $1 \%$ of total costs [1], so HTS technologies are expected to be considerably cheaper in future.

Now, superconducting devices for power engineering (i.e. cables, transformers, generators) are of great interest in those power systems where maximum current density with minimum mass and dimensions parameters is required (i.e. transport, marine, aviation power systems, and so on). On the other hand, traditional power engineering often uses so-called "current limiting devices" that allow very fast (less than $1 \mathrm{~ms}$ ) short circuit current limitation without inserting additional reactance to the system [2]-[5]. A power transformer can be used as a current limiting device when its windings are assembled of superconductors, that is undeniable advantage over traditional transformers. In addition, a superconducting transformer (SCT) has other advantages over common transformers of the same power: explosion and fire 
safety, lower mass and dimensional parameters, possibility of continuous double overloading, reduced short circuit losses.

However, adding the possibility of short circuit current limitation into a transformer specifies requirements to the transformer construction caused by the features of existing regimes of the power system. For example, SCT operation during successful automatic reclosing is of interest. In this case, superconducting wires are overheated due to short circuit conditions with the initial short circuit current limitation due to the rise of superconducting wire resistance. The following problem will be discussed in the paper: which combinations of SCT winding parameters are required for the process of heating at current limitation that it does not result in failures of normal operation of the transformer after successful short circuit clearing in the automatic reclosing cycle.

The present study is performed with the help of the mathematical model for the process of transformer winding overheating under short circuit conditions in the circuit based on the results of the fullscale experiment on a small superconducting coil made of HTS wire of the 2nd generation produced by SuperOx ${ }^{\mathrm{TM}}$ [6]. It is assumed that processes in the coil, which represents a real transformer in a first approximation, will be similar to processes in a large SCT. For that reason, terms "coil" and "transformer" used in the paper will be equivalent.

\section{PROBLEM DEFINITION}

The starting point of the study is the investigation of the current limitation process with the use of a coil made of a HTS tape (SuperOx ${ }^{\mathrm{TM}}$ ) having the length of $3 \mathrm{~m}$, width of $4 \mathrm{~mm}$ and critical current of $80 \mathrm{~A}$ (see Figure 1).

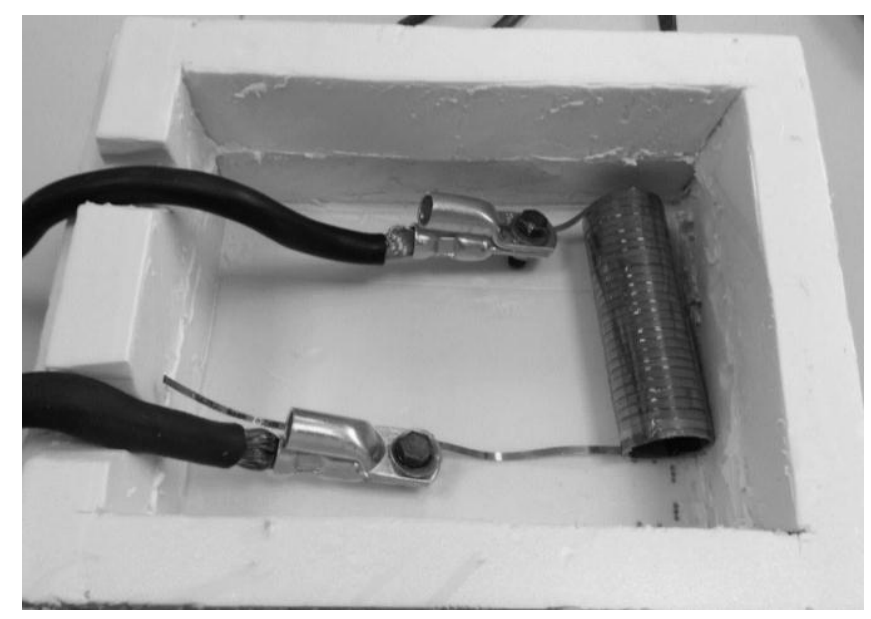

Figure 1. HTS coil connected to the power source in the polystyrene bath for liquid nitrogen filling

The full test circuit is illustrated in Figure 2. The circuit includes the load assembled from nichrome wires and the short-circuit switch, which is represented by a common automatic circuit breaker. The oscillogram of current in the moment of short circuit was recorded with the use of a current clamp (see Figure 3).

The curve of current change versus time is shown as a black solid line in the oscillogram. The noisy curve illustrates voltage drop at the coil. Curve distortion is caused by low resolution of voltage measuring by FLUKE 435-II. Figure 3 shows that the current curve has some distortion caused by nonlinearity of the current-voltage characteristic (the I-V curve) of the superconducting coil. The maximum value of current reaches $132 \mathrm{~A}$ with limitation and $175 \mathrm{~A}$ without limitation. The conducted full-scale experiment and obtained information can be used for the development of a mathematical model for a transient process in the case of short circuit at the load side.

The oscillogram of limited current for various HTS current limiting devices given in Figure 4 [7] is different from the oscillogram obtained during our experiment (see Figure 3). There are no any distortions after the first half-cycle, then the signal becomes sinusoidal. It can be explained in the following way. Current limiting devices are designed in such a manner that flowing of high values of short circuit currents causes "jumping" over the nonlinear area of the I-V curve of a superconducting wire, then steady state 
conditions of short circuit will be fully determined by resistances of resistive materials of a current limiting device (for example, copper of a superconducting tape). However, the present study is devoted to investigations of thermal operating regimes of the superconducting coil in the nonlinear area of the superconductor I-V curve.

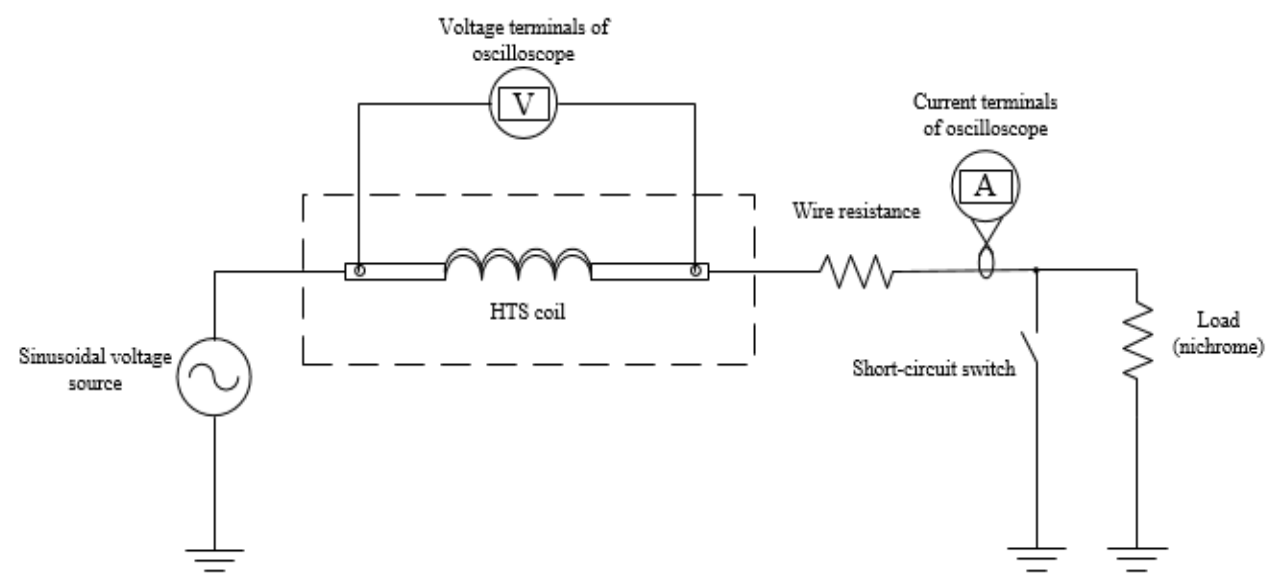

Figure 2. Test circuit

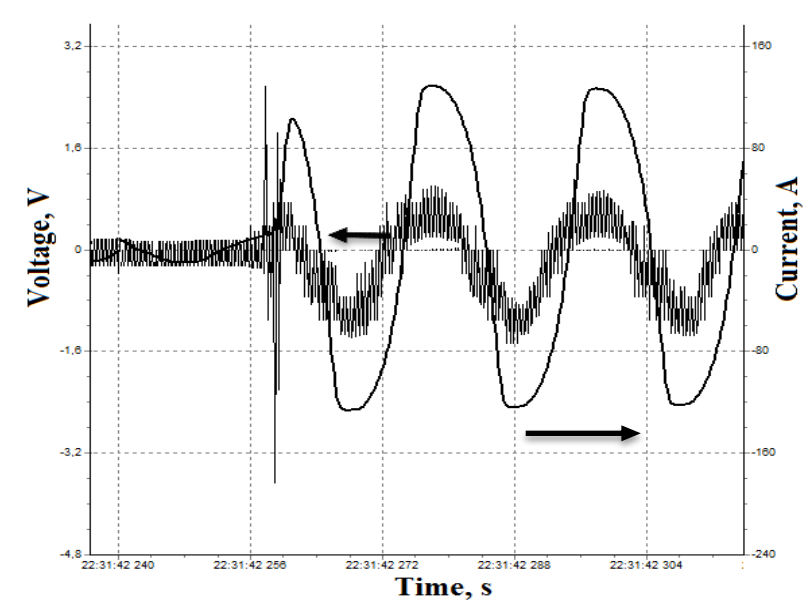

Figure 3. Oscillogram of transient process at short circuit on the load side

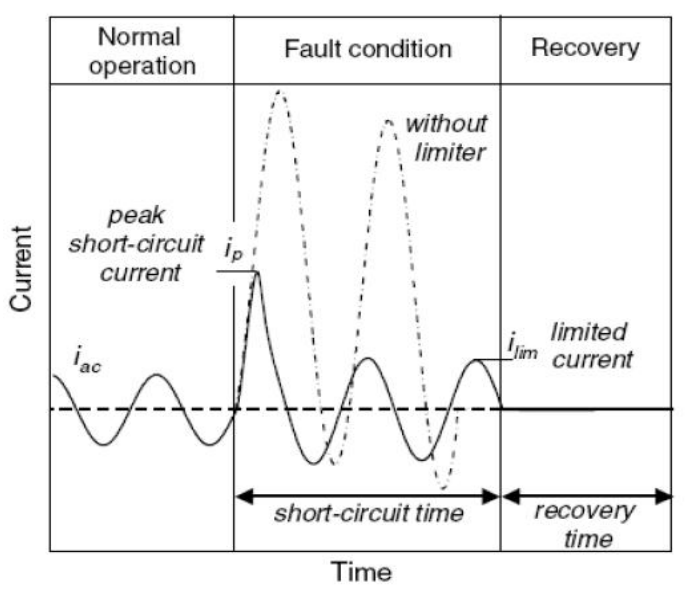

Figure 4. Operating regimes of a current limiting device circuit:

The circuit given in Figure 2 can be described by a simple differential equation for an inductive

$$
L \frac{d i}{d t}+i\left(R_{\text {sup.c }}(I, T)+R\right)=U \sin (\omega t)
$$

where $\mathrm{L}$ - inductance of the superconducting coil, $\mathrm{H} ; \mathrm{i}$ - current in the circuit, A; U - source voltage, V; R load resistance or short circuit resistance, depending on the considered regime, Ohm; $\mathrm{R}_{\text {sup.c }}-$ resistance of the superconducting coil, Ohm.

Nonlinear character of superconducting coil resistance should be considered in the equation. It depends on the modulus of instantaneous current in accordance with the following equation derived by transforming the I-V curve expression for a superconducting tape (by division of equation parts by current):

$$
R_{\text {sup.c }}(I)=\frac{E_{0}}{i}\left(\frac{i}{I_{c}}\right)^{n},
$$


where $I_{c}$ - current, which is conventionally chosen as a critical current for a superconductor, A. As a rule, current is considered to be critical if electric field strength in a superconductor at this current is equal to 1 $\mu \mathrm{V} / \mathrm{cm}$ (this criterion is proposed by a HTS tape manufacturer); $\mathrm{E}_{0}$ - voltage at the coil tape at critical current flowing, $\mathrm{V} ; \mathrm{n}$ - exponent of the I-V curve of the superconducting tape. At the same time, a critical current $I_{c}$ from (2) is determined by the following equation [8]:

$$
I_{c}(T)=-\frac{I_{c 0}}{0,1848} \ln \left(\frac{T}{93}\right)
$$

where $\mathrm{I}_{\mathrm{c} 0}$ - critical current in a self-field at $77 \mathrm{~K}, \mathrm{~A} ; \mathrm{T}-$ ambient temperature, $\mathrm{K}$. It should be noted that a critical current is considered to be equal to zero at temperatures above $90 \mathrm{~K}$. Equation (3) shows that the process of winding heating should be taken into account when developing an adequate model of current limitation. To consider the heating process of the superconducting coil, the heat balance equation can be formulated:

$$
\frac{d T}{d t}=\frac{Q\left(I, R_{\text {Sup.c }}\right)-A q(\Delta T)}{C_{\Sigma}(T)}
$$

where $\mathrm{Q}$ - the quantity of heat released in the coil passing into the resistive state at current flowing per unit time, W; A - surface area of the coil, $\mathrm{m}^{2}$; $\mathrm{q}$ - density of heat flow passing into liquid nitrogen from the coil surface per unit time, $\mathrm{W} / \mathrm{m}^{2} ; \mathrm{C}_{\Sigma}-$ total heat capacity of the HTS coil depending on the temperature, J/K. The quantity of heat produced during current flowing through the HTS coil can be calculated as:

$$
Q\left(I, R_{\text {sup.c }}\right)=\int_{t_{\text {sc }}}^{t_{\text {clear }}} i\left(R_{\text {sup.c }}\right)^{2} R_{\text {sup.c }}(T) d t
$$

where $t_{s c}$ and $t_{\text {clear }}$ - time of occurrence and time of clearance of a short circuit respectively.

Density of heat flow $\mathrm{q}$ has a complex dependence on the temperature difference between a cooling surface and liquid nitrogen that is illustrated in Figure 5. As is shown, if the superconducting coil is considerably overheated, the process of heat removal will be ineffective, that will cause further overheating up to HTS wire burnout.

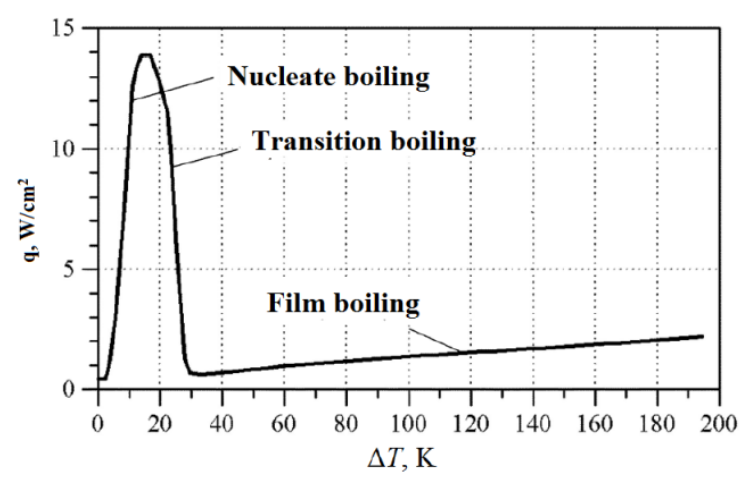

Figure 5. Heat flow density versus the temperature difference between a cooling surface and liquid nitrogen [9]

Equations (1) to (5) need to be combined into the system of equations to be solved simultaneously. This solution can be performed numerically in Matlab Simulink. As stated before, using the proposed model, it is necessary to determine with which combinations of regime and design parameters a superconducting transformer can be operated well after the successful automatic reclosing cycle. In other words, it is necessary to find such impermissible values of parameters for overheating of transformer windings that a superconducting transformer does not return into the superconducting state after successful automatic reclosing. It means that a winding, being heated during the transition into the normal state, could not be cooled within the automatic reclosing cycle.

The present study aims at showing rather qualitative character of thermal processes in the HTS device, because the developed mathematical model has been verified previously by only a current behaviour 
in the circuit with the small superconducting coil. However, some of obtained results are supposed to be generalized to more complex devices as superconducting transformers.

The combinations of such parameters as a short circuit duration and a dead-time period determine conditions for a transformer winding to be cooled enough after overheating by short circuit currents, then a transformer can return into the superconducting state.

An exponent of the I-V curve of the superconducting tape has the deeper meaning. An exponent $n$ of the current-voltage characteristic of the superconductor determines the level of short circuit current limitation that, evidently, will influence directly on the value of overheating. As was shown in [10], the nonlinearity parameter of the I-V curve generally will be different in different parts of the superconducting device (e.g. the coil). This fact is not considered in the study. The nonlinearity parameter of the I-V curve is assumed to be of the average value along the superconducting wire. The necessity of varying values of this parameter is determined by the hypothesis that the exponent of the I-V curve will be changed in the result of mechanical and thermomagnetic impacts on the superconductor, thus changing the form of a current signal and, consequently, a value of heating.

\section{INFLUENCE OF THE NONLINEARITY PARAMETER OF THE I-V CURVE ON THE LEVEL OF CURRENT LIMITATION}

The aim of this section is to demonstrate that the nonlinearity parameter of the I-V curve has a considerable influence on the level of current limitation.

The circuit given in Figure 2 can be reduced to the classical form of the single line diagram (Figure 6). Elements of the equivalent circuit have real values determined in the physical experiment described above. Circuit parameters and some calculated values are given in Table 1.

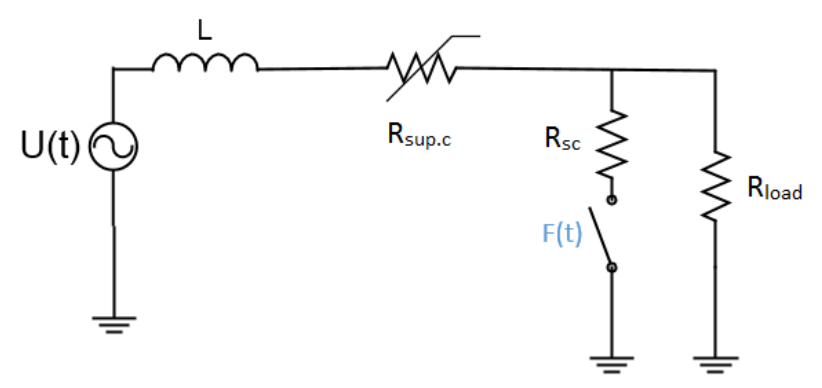

Figure 6. Single line equivalent circuit for simulation of short circuit at the load side

Table 1. Circuit Parameters and some Calculated Values

\begin{tabular}{cc}
\hline \multicolumn{2}{c}{ Circuit parameters } \\
\hline Source voltage amplitude $\mathrm{U}_{\max }$ & $5 \mathrm{~V}$ \\
Frequency of power supply voltage & $50 \mathrm{~Hz}$ \\
Inductance L & $8 \mu \mathrm{H}$ \\
Load resistance $\mathrm{R}_{\text {load }}$ & $0.45 \mathrm{Ohm}$ \\
Short circuit resistance $\mathrm{R}_{\mathrm{sc}}$ & $0.029 \mathrm{Ohm}$ \\
Calculated parameters & $11.1 \mathrm{~A}$ \\
Current amplitude under normal conditions $\mathrm{I}_{\text {load }}$ & $175 \mathrm{~A}$ \\
Short circuit current amplitude (without limitation) $\mathrm{I}_{\mathrm{sc}}$ & 15.8 \\
\hline $\mathrm{I}_{\mathrm{sc}} / \mathrm{I}_{\mathrm{load}}$ & \\
\hline
\end{tabular}

Solution of the system of Equations (1) to (5) with $\mathrm{F}(\mathrm{t})$ switch closing and with real test circuit parameters gives the following current oscillogram of the transient process shown in Figure 7.

Figure 7 shows good coincidence between the curve of limited current and the experimentally obtained curve that implicitly demonstrates the adequacy of the model. It is observed from the presented oscillogram that current is limited from $175 \mathrm{~A}$ to $132 \mathrm{~A}$ (or the ratio $\mathrm{I}_{\mathrm{sc}} / \mathrm{I}_{\text {load }}$ decreases from 15.8 to 11.9) that corresponds to the exponent $n$ (see Equation (2)) being equal to 15.5. It should be noted that if unlimited and limited current is known, under all other conditions being equal, the average $n$ can be determined for any device made of HTS wire. Then, the dependence of the maximum current in the circuit on the $n$ parameter will be considered (Figure 8). 


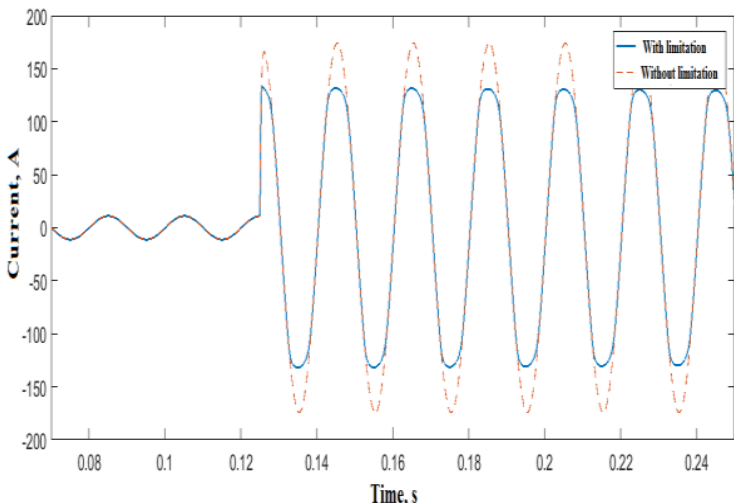

Figure 7. Oscillogram of short circuit current with and without current limitation

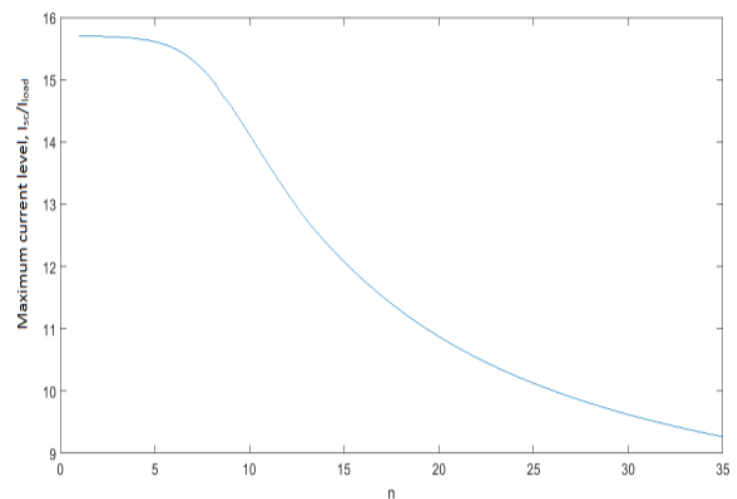

Figure 8. Maximum limited short circuit current level versus the nonlinearity parameter of the I-V curve

Under real experimental conditions, the $n$ parameter for HTS wires does not usually exceed 15 (being more than 30 for low-temperature superconducting wires) [11]. The curve given in Figure 8 shows insignificant current limitation at low values of $n$. This fact is important because, as was stated above, thermomagnetic and mechanical impacts may change the exponent $n$ of the device made of HTS wires. If the average $n$ of the I-V curve for this device is low, the device will be inefficient in current limitation.

Then, consider the curve of the dependence of maximum overheating on the exponent $n$ for the specified case (Figure 9). However, it should be noted that the curve of maximum overheating has a considerable dependence on circuit parameters, such as short circuit resistance and HTS device resistance in the normal state. Nevertheless, the obtained curve demonstrates the explicit dependence of overheating on nonlinearity of the I-V curve. This fact will be considered in detail below.

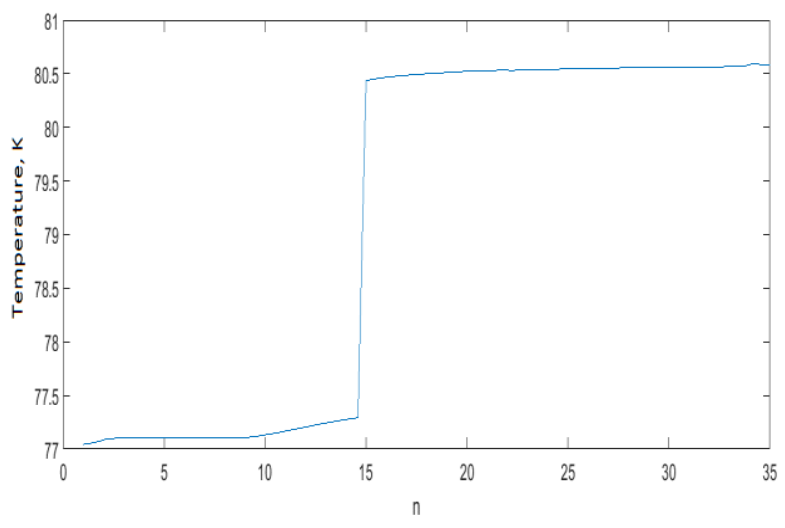

Figure 9. Maximum temperature of HTS device heating versus the nonlinearity parameter of the I-V curve

\section{INFLUENCE OF THE DEAD-TIME PERIOD AFTER SHORT CIRCUIT CLEARING ON THE DEGREE OF COOLING FOR A HTS DEVICE}

Since a HTS transformer is intended to be used in real power systems, then its operation should not result in the loss of power system stability. One of such regulated regimes is the cycle of automatic reclosing which includes a dead-time period required for self-clearance of short circuit at a power transmission line. According to [12], a time setting for the first cycle of automatic reclosing in $6-10 \mathrm{kV}$ networks is usually set to not more than $2 \mathrm{~s}$. The minimum setting for these networks varies in the wide range and depends on many factors. As a first approximation, the minimum setting is assumed to be $0.4 \mathrm{~s}$.

Since the process of heat release from a winding surface is stopped in a dead-time period, a temperature change will be fully determined by heat removal into a cooling liquid that depends on the characteristic shown in Figure 5. Transformer cooling does not also depend on a method of preliminary heating. In other words, it does not depend on short circuit resistance, source voltage and other parameters. 
Therefore, the task is to determine the minimum temperature for cooling of the superconducting coil depending on the maximum temperature of preliminary heating resulting from short circuit current flowing. Simulation of these processes will be also performed with the use of the circuit presented in Figure 6 with the parameters from Table 1 except the short circuit resistance, $\mathrm{R}_{\mathrm{sc}}$, now being equal to zero. Zero short circuit resistance gives the regime with the maximum short circuit current, and, consequently, with the maximum coil heating.

The results of simulation are presented in Figure 10 to Figure 13. Figure 10 and Figure 11 show the characteristics of temperature versus time with the following sequence of events:

Time $\mathrm{t}=0 . .125 \mathrm{~ms}-$ normal regime;

$125 . .325 \mathrm{~ms}$ - short circuit;

$325 . .725 \mathrm{~ms}$ - short circuit clearance and the dead-time period of the automatic reclosing cycle;

725..1000 ms - successful automatic reclosing and return to steady state conditions.

Figure 10 illustrates the case of successful thermal return of the superconducting coil into the superconducting state after $0.4 \mathrm{~s}$ of the dead-time period, and the characteristic of heat flow versus time. Figure 11 shows the case of NOT RETURNING of the coil into the superconducting state.

Therefore, the question has arisen: which factors influences on the process of returning or not returning of a supercoducting device into the superconducting state after overheating? It is evident to assume that in the case of the constant dead-time period the success of thermal returning will depend on the temperature reached during short circuit. In other words, it is necessary for the device to be cooled below the specific temperature within the dead-time period. This temperature will be determined by a current amplitude of the further steady state regime in the case of successful automatic reclosing. If it is assumed that a current amplitude in the steady state regime does not exceed 40 A (with the critical current of the HTS coil of 80 A), then using this value in (3) and deriving an unknown $\mathrm{T}$, the required temperature for thermal returning can be found, being equal to $85 \mathrm{~K}$.
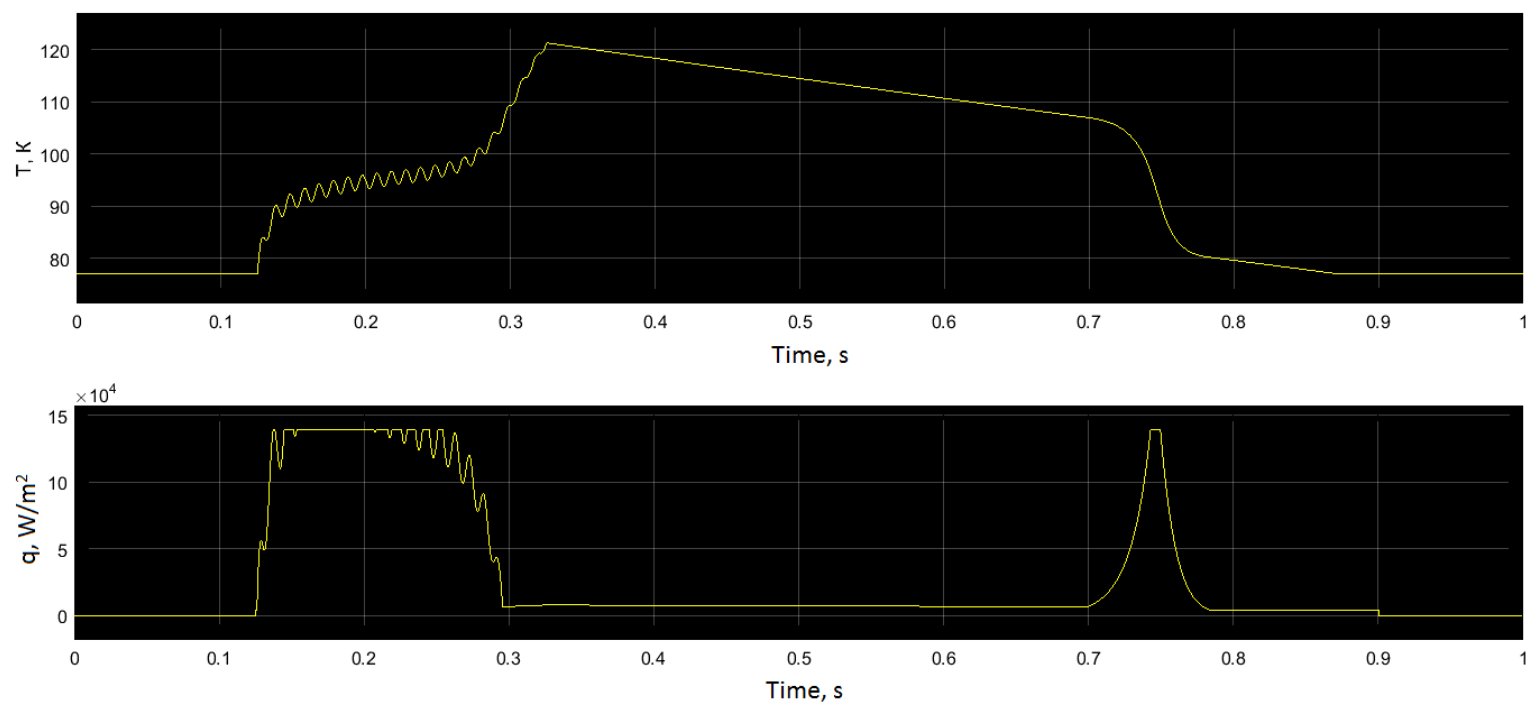

Figure 10. Characteristics of temperature $(T)$ and heat flow density (q) versus time; thermal returning is observed

Figure 12 and Figure 13 present characteristics of the minimum temperature reached in the deadtime period versus the maximum temperature reached under the previous short circuit conditions. Figure 12 has the characteristic for the dead-time period of $t_{d t}=0.4 \mathrm{~s}$, while Figure $13-$ for $t_{d t}=2 \mathrm{~s}$. These cases consider two variants of the tape length in the HTS coil being equal to $3 \mathrm{~m}$ and $6 \mathrm{~m}$.

The temperature limit required for thermal returning of the coil, made of the tape with the length of $3 \mathrm{~m}$, is marked with the black solid line in figures. As is shown, it is important for thermal returning of the coil that the temperature of the previous regime (short circuit) does not exceed $100 \mathrm{~K}$ at the dead-time period of $0.4 \mathrm{~s}$ and $193 \mathrm{~K}$ at the dead-time period of $2 \mathrm{~s}$. These results are planned to be verified experimentally on the real HTS coil shown in Figure 1. 

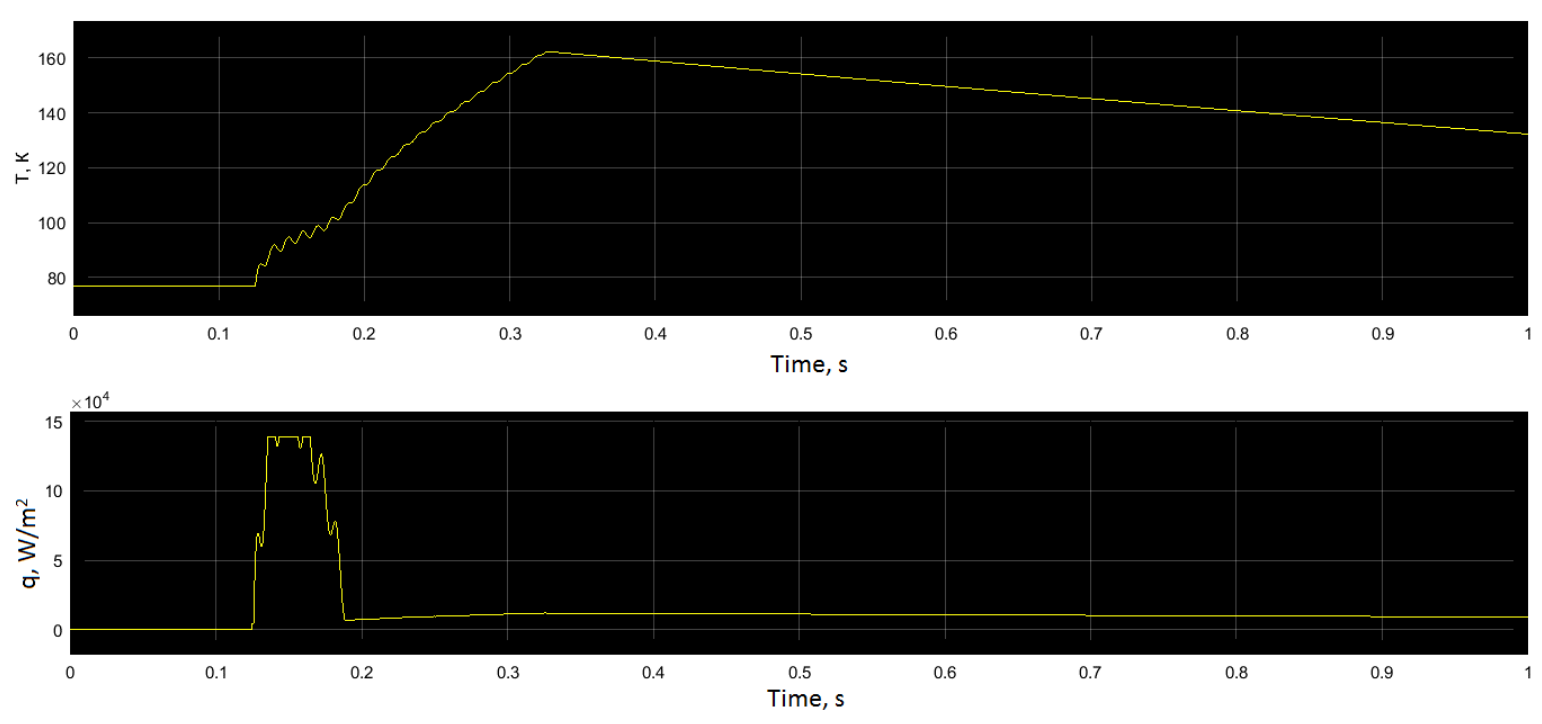

Figure 11. Characteristics of temperature $(\mathrm{T})$ and heat flow density $(\mathrm{q})$ versus time; thermal returning is not observed

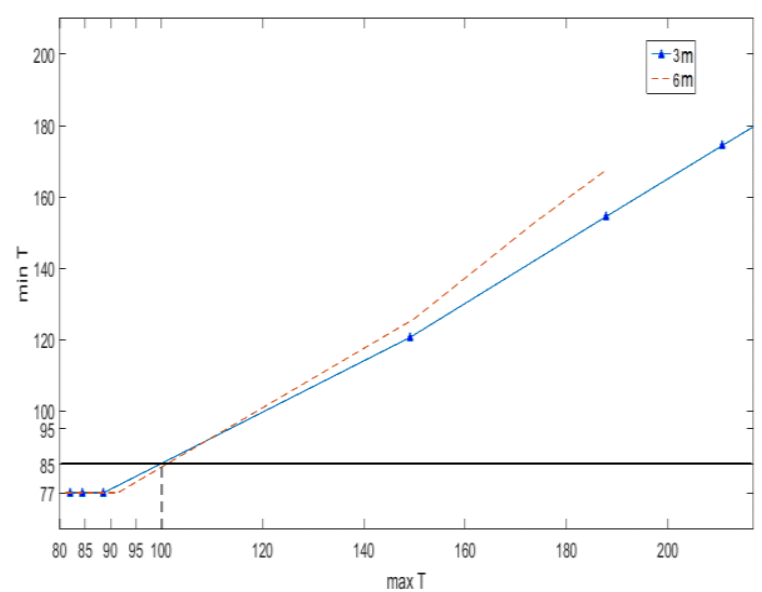

Figure 12. Characteristic of the minimum temperature of cooling in the dead-time period versus the maximum temperature of the preliminary winding heating at $\mathrm{t}_{\mathrm{dt}}=0.4 \mathrm{~s}$

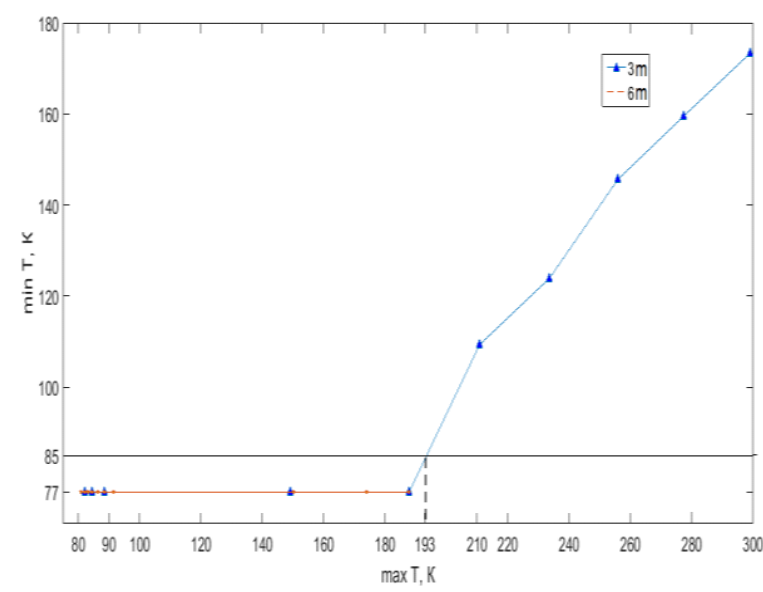

Figure 13. Characteristic of the minimum temperature of cooling in the dead-time period versus the maximum temperature of the preliminary winding heating at $t_{d t}=2 \mathrm{~s}$

For comparison, Figure 12 also shows the characteristic for the coil with the tape of $6 \mathrm{~m}$, which is heated slowly and cooled slowly, that is correct because the total weight of the HTS tape and, consequently, heat capacity increases. This fact may have a positive aspect, as well as a negative aspect. On the one hand, a large HTS device is heated slowly by currents exceeding the critical. On the other hand, it is also cooled slowly after current interruption. Therefore, there is a problem of optimizing processes of heating and cooling for large HTS devices, such as transformers. Discussion and solution of this optimization problem is planned in further investigations.

\section{INFLUENCE OF DIFFERENT PARAMETERS OF THE HTS DEVICE ON THE DEGREE OF OVERHEATING}

This section considers the degree of overheating for the HTS coil with variation of different parameters of the coil and the circuit (Figure 6), and regime parameters, such as the short circuit duration. The study will be performed by the series of calculation experiments with variation of the given parameters. 


\subsection{Experiment 1 - Dependence of the maximum temperature of HTS coil overheating on the exponent of the $\mathrm{I}-\mathrm{V}$ curve and the short circuit duration}

Figure 14 presents the series of surfaces representing the dependence of the maximum temperature of coil overheating on the $n$ parameter of the I-V curve (Equation (2)), the short circuit duration, and the short circuit resistance. Other parameters of the circuit are presented in Table 2.

Table 2. Equivalent Circuit Parameters for Experiment 1

\begin{tabular}{cc}
\hline Source voltage amplitude $\mathrm{U}_{\max }$ & $5 \mathrm{~V}$ \\
Frequency of power supply voltage & $50 \mathrm{~Hz}$ \\
Inductance L & $8 \mu \mathrm{H}$ \\
Load resistance $\mathrm{R}_{\text {load }}$ & $0.45 \mathrm{Ohm}$ \\
Short circuit resistance $\mathrm{R}_{\mathrm{sc}}$ & $0.0001 \ldots 0.0286 \mathrm{Ohm}$ \\
Short circuit duration, $\mathrm{t}_{\mathrm{sc}}$ & $0.1 . .0 .2 \mathrm{~s}$ \\
Nonlinearity parameter of the I-V curve, $\mathrm{n}$ & $1 . .20$ \\
Tape length of the HTS coil, $\mathrm{m}$ & 3 \\
\hline
\end{tabular}

Surfaces from Figure 14 are structured in a special way to demonstrate some considerable effects. Firstly, the maximum temperature has a weak dependence on the short circuit duration almost at all surfaces becoming stronger at last two surfaces $\left(R_{s c}=0.02 \mathrm{Ohm}\right.$ and $\left.R_{s c}=0.0286 \mathrm{Ohm}\right)$. This is explained by the fact that a time constant of superconducting coil heating is smaller than the short circuit duration. When short circuit resistance increases, current in the circuit begins decreasing. Then, energy for coil heating decreases proportionally to the squared current, thus increasing a time constant for coil heating that determines the dependence of the maximum temperature on the short circuit duration.
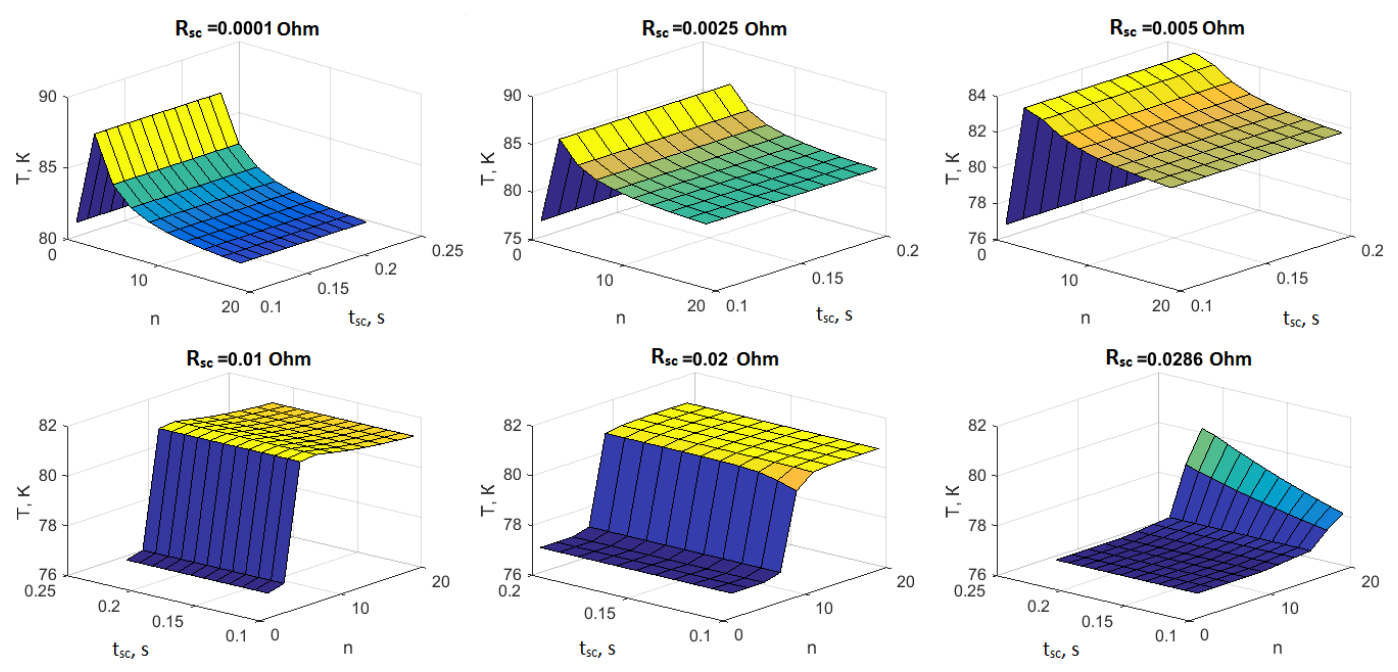

Figure 14. Characteristic of the maximum temperature of HTS coil overheating versus the exponent of the I-V curve $(n)$ and the short circuit duration $\left(t_{s c}\right)$ with various short circuit resistances $\left(R_{s c}\right)$

Secondly, the top graphs in Figure 13 illustrate the evident extremum of the maximum temperature depending on the $n$ parameter. This extremum is caused by complex interrelation between the current in the circuit and the HTS coil resistance influencing each other. Their combination with the specified $n$ results in the maximum energy release from the HTS wire surface. The bottom graphs in Figure 14 show that the temperature maximum is not explicitly observed. It is due to the short circuit resistance being commensurable with the coil resistance in the resistive state. It is important to note that this maximum is shifted to the area of higher $n$ with the increase of the short circuit resistance.

Thirdly, it should be noted that each surface has the area of sharp temperature increase after the specified $n$. If the nonlinearity parameter of the I-V curve is fixed, for example, at the level experimentally obtained $(n=15.5)$, the dependence of maximum temperatures on two other parameters will be as shown in Figure 15. It can be seen that the short circuit duration at low values of $R_{\mathrm{sc}}$ does not have a great influence on the maximum temperature, because steady-state thermal conditions are reached much faster than relay 
protection operates. At high values of short circuit resistance short circuit currents are not sufficient to cause considerable overheating.

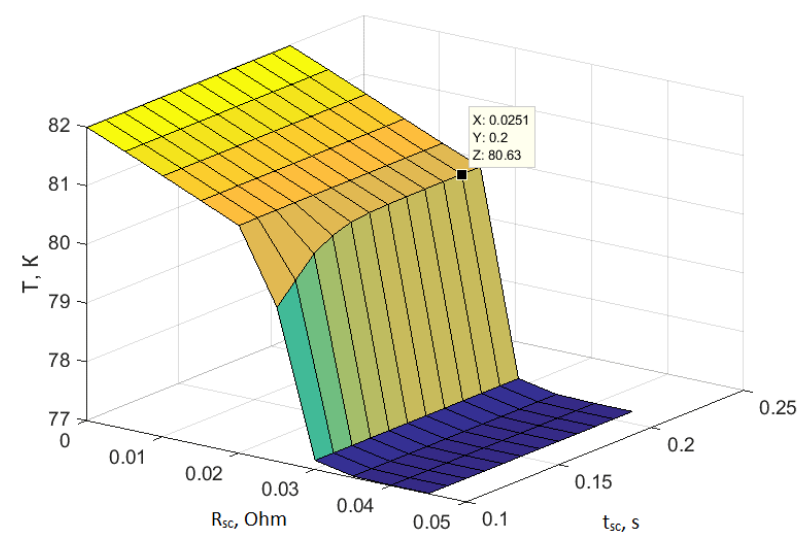

Figure 15. Characteristic of the maximum temperature of HTS coil overheating versus the short circuit duration $\left(\mathrm{t}_{\mathrm{sc}}\right)$ and the short circuit resistance $\left(\mathrm{R}_{\mathrm{sc}}\right)$ at $\mathrm{n}=15.5$

However, explanations given above are correct under conditions of the real experiment for the small coil. As for large devices, it is expected that the time constant will have greater influence on the level of maximum temperatures, because larger devices are difficult to be heated. In addition, short circuit currents in large devices are considerably higher.

\subsection{Experiment 2 - Dependence of the maximum temperature of HTS coil overheating on the exponent of the I-V curve and the source voltage}

Consider coil behaviour under intentional overheating by high values of power taking into account the nonlinearity parameter of the I-V curve. Regulation of the source power is performed by the change of its voltage. Circuit parameters and regime parameters are given in Table 3. Simulation results in the form of the surface are shown in Figure 16.

The plotted surface represents the importance of high values of $n$ for HTS devices (in the range of $5 . .15$ ), because the temperature increase in the case of the voltage rise becomes critical at low values of $n$ (lower than 5). This assumption should be verified at the model of a large HTS device. However, undoubtedly, heat release will be maximum at low values of the nonlinearity parameter of the I-V curve due to not so fast current limitation with rather fast increase of resistance.

Table 3. Equivalent Circuit Parameters for Experiment 2

\begin{tabular}{cc}
\hline Source voltage amplitude $\mathrm{U}_{\max }$ & $10 . .25 \mathrm{~V}$ \\
Frequency of power supply voltage & $50 \mathrm{~Hz}$ \\
Inductance $\mathrm{L}$ & $8 \mu \mathrm{H}$ \\
Load resistance $\mathrm{R}_{\mathrm{load}}$ & $0.45 \mathrm{Ohm}$ \\
Short circuit resistance $\mathrm{R}_{\mathrm{sc}}$ & $0.0001 \mathrm{Ohm}$ \\
Short circuit duration, $\mathrm{t}_{\mathrm{sc}}$ & $0.2 \mathrm{~s}$ \\
Nonlinearity parameter of the I-V curve, $\mathrm{n}$ & $1 . .20$ \\
Tape length of the HTS coil, $\mathrm{m}$ & 3 \\
\hline
\end{tabular}




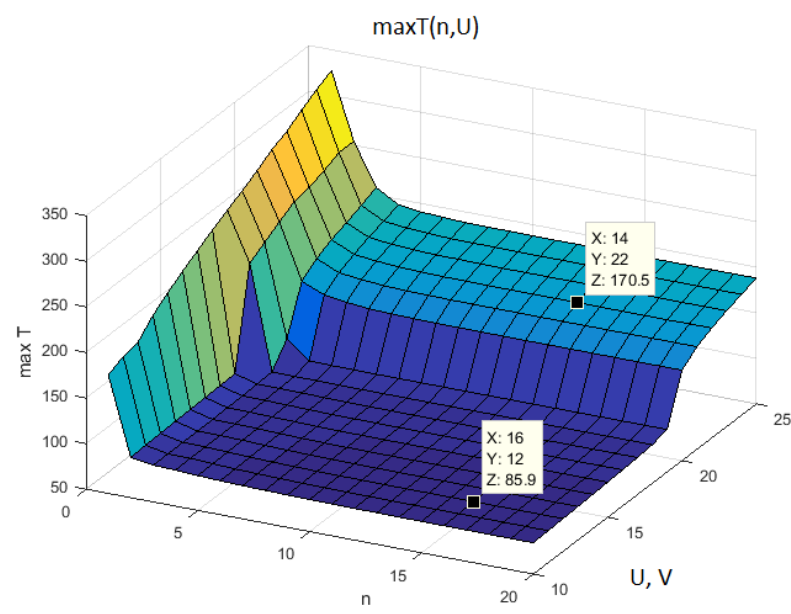

Figure 16. Characteristic of the maximum temperature of HTS coil overheating versus the nonlinearity parameter of the I-V curve and the source voltage

\section{CONCLUSION}

Using the physical model of current limitation with the use of the coil made of superconducting wire SuperOx ${ }^{\mathrm{TM}}$, the mathematical model of the transient process in the nonlinear active-inductive circuit with consideration of thermal heating was developed on the basis of two differential (1 and 4) and three algebraic $(2,3$ and 5$)$ equations.

Based on the developed model, different aspects of thermal behaviour of the superconducting coil with variation of its parameters and regime parameters in the wide range were studied. The following conclusions can be made:

a. As shown in Section 3, based on known values of limited and NOT limited short circuit current, the average nonlinearity parameter of the I-V curve can be uniquely determined for a superconducting device;

b. It was shown that the level of current limitation considerably increases with the rise of the nonlinearity parameter of the I-V curve;

c. As shown in Section 4, the possibility for returning an HTS device into the superconducting state in the dead-time period of the automatic reclosing cycle is fully determined by cooling conditions and the temperature reached under the previous short circuit regime;

d. The criterion for determination of the permissible temperature of overheating was proposed. It is based on the current level in the further steady state regime (with assumption of successful automatic reclosing in the first cycle) with the fixed dead-time period;

e. Direct influence of the nonlinearity parameter of the I-V curve on the level of maximum temperatures of HTS device overheating was found: at low values of $n$, the combination of insufficient current limitation and sufficiently increased resistance results in the maximum heat release in the HTS device. This allows making an assumption that HTS devices with low average nonlinearity parameters of the I-V curve should not be used for short circuit current limitation that needs to be verified by a physical experiment.

For further investigations, it is planned to adjust the proposed model by full-scale experiments, with the consideration of the temperature change in HTS devices caused by current flowing exceeding critical values. Statement and solution of the optimization problem is also planned based on conclusions given in Section 3: which combination of the dead-time period and the maximum overheating temperature is sufficient for returning an HTS device into the superconducting state.

\section{ACKNOWLEDGEMENTS}

The research was carried out under the State Assignment of the Ministry of Education and Science of the Russian Federation, Project 8.6809.2017/8.9.

\section{REFERENCES}

[1] Amelichev V. A., "Methods of manufacture of the 2d generation HTS wire: Brief overview," Global scientific potential, 2012. 
[2] Fisher L. M., et al., "Superconducting fault current limiter for railway transport," Physics of Atomic Nuclei, vol. 78, no. 14, pp. 1654-1657, 2015.

[3] Luongo C. A., et al., "Next generation more-electric aircraft: a potential application for HTS superconductors," IEEE Transactions on Applied Superconductivity, vol. 19, no. 3, pp. 1055-1068, 2009.

[4] Manusov V. Z., et al., "A Study of Superconducting Transformer with Short-Circuit Current Limitation," International Journal of Electrical and Computer Engineering (IJECE), vol. 8, no. 1, 2018.

[5] Murukeshan A. and Rajasekaran R., "Power system stability enhancement and improvement of LVRT capability of a DFIG based wind power system by using SMES and SFCL," International Journal of Electrical and Computer Engineering, vol. 3, no. 5, pp. 618, 2013.

[6] Lee S., et al., "Development and production of second generation high Tc superconducting tapes at SuperOx and first tests of model cables," Superconductor Science and Technology, vol. 27, no. 4, 044022, 2014.

[7] Wojtasiewicz G., "Fault Current Limitation by 2G HTS Superconducting Transformer-Experimental Investigation," Acta Physica Polonica A., vol. 130, no. 2, pp. 516-520, 2016.

[8] Manusov V. Z. and Aleksandrov N. V., "Limitation of short circuit currents with the use of transformers with high temperature superconducting windings," Bulletin of the Tomsk Polytechnic University, vol. 323, no. 4, 2013.

[9] Merte H. and Clark J. A., "Boiling heat transfer data for liquid nitrogen at standard and near-zero gravity," Advanced Cryogenic Engineering, vol. 7, pp. 546-550, 1962.

[10] Ilyin Y. A., et al., "Critical Current Distribution along the BI-2212 High Temperature Superconducting Coil," Advances in Superconductivity X, Springer, Tokyo, pp. 799-802, 1998.

[11] Mints R. G. and Rakhmanov A. L., Rev. Mod. Phys., vol. 53, pp. 551-560, 1981.

[12] Ovchinnikov V. V., “Automatic reclosing,” Moscow, STC “Energoprogress,” 2001.

\section{BIOGRAPHIES OF AUTHORS}

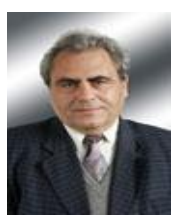

Vadim Z. Manusov. Novosibirsk State Technical University, Department of Power Supply Systems, D. Eng, Professor. Main directions of investigations: methods of artificial intelligence for planning and optimization of operating regimes of power systems.

E-mail: manusov@corp.nstu.ru

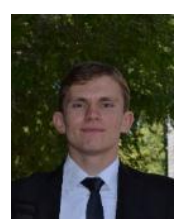

Dmitry O. Krjukov. Novosibirsk State Technical University, Department of Power Supply Systems, student, Assistant of the department. Main directions of investigations: Application of superconductors in the electric power industry

E-mail: fire-paladin@mail.ru 